\title{
Environment-Sensitive Thermal Coarsening of Nanoporous Gold
}

\author{
Satoko Kuwano-Nakatani ${ }^{1, *}$, Takeshi Fujita ${ }^{2}$, Kazuki Uchisawa ${ }^{1}$, Daichi Umetsu ${ }^{1}$, \\ Yu Kase ${ }^{1}$, Yusuke Kowata ${ }^{1}$, Katsuhiko Chiba ${ }^{1}$, Tomoharu Tokunaga ${ }^{3}$, \\ Shigeo $\mathrm{Arai}^{3}$, Yuta Yamamoto ${ }^{3}$, Nobuo Tanaka ${ }^{3}$ and Mingwei Chen ${ }^{2,4}$ \\ ${ }^{1}$ Faculty of Engineering, Tohoku Gakuin University, Tagajo 985-8537, Japan \\ ${ }^{2}$ Advanced Institute for Materials Research, Tohoku University, Sendai 980-8577, Japan \\ ${ }^{3}$ Ecotopia Science Institute, Nagoya University, Nagoya 464-8603, Japan \\ ${ }^{4}$ State Key Laboratory of Metal Matrix Composites, School of Materials Science and Engineering, \\ Shanghai Jiao Tong University, Shanghai 200030, P. R. China
}

In order to investigate environmental effects on the ligament/pore coarsening of nanoporous gold (NPG), we studied the thermal coarsening of NPG both in air and vacuum by ex situ observation, and found that it has high structural stability against heat treatment in vacuum. To clarify the nature of this phenomenon, we investigated the thermal coarsening of NPG by in situ environmental transmission electron microscopy. At an elevated temperature $\left(400^{\circ} \mathrm{C}\right)$, the coarsening of ligaments/pores was triggered by introducing either pure $\mathrm{N}_{2}$ or $\mathrm{O}_{2}$ gas into the transmission electron microscopy (TEM) chamber (but not by Ar gas). We thus conclude with a discussion on the mechanism for thermal coarsening of NPG. [doi:10.2320/matertrans.MF201403]

(Received October 1, 2014; Accepted November 11, 2014; Published December 27, 2014)

Keywords: nanoporous gold, environmental factors, thermal coarsening, catalytic materials, environmental transmission electron microscopy

\section{Introduction}

Controlling the size of nanomaterials is essential to tailoring their functional properties for optical and catalytic applications. Particularly, nanoporous gold (NPG) - made by dealloying a gold/silver alloy — has attracted considerable attention because of its versatile optical ${ }^{1)}$ and catalytic properties $^{2,3)}$ in a wide range of applications. NPG has a bicontinuous structure of ligaments/nanopores whose size can be tuned from $5 \mathrm{~nm}$ to several tens of microns by altering dealloying conditions (dealloying time and temperature) and subsequent thermal coarsening. ${ }^{4-6)}$

As with gold nanoparticle/oxide systems, nanoporous gold is catalytically active for $\mathrm{CO}$ oxidation at room temperature. ${ }^{1)}$ The geometrically required surface curvature provides abundant active sites composed of surface defects such as kinks and steps. ${ }^{7}$ The ligament/pore size influences the density of such surface defects, which affects chemical reactivity. Therefore, it is important to understand the factors that influence coarsening behavior.

In this study, we investigated the thermal coarsening of NPG both in air and vacuum by ex situ observation, and found that NPG has an inherently high structural stability against heat treatment in vacuum. To clarify the nature of this phenomenon, we investigated thermal coarsening by in situ environmental transmission electron microscopy (ETEM). Four different gas environments were selected for annealing, i.e. vacuum, $\mathrm{N}_{2}, \mathrm{O}_{2}$, and Ar. We found that while NPG has high structural stability in vacuum at an elevated temperature $\left(400^{\circ} \mathrm{C}\right)$, the coarsening of ligament/pores was triggered by introducing either pure $\mathrm{N}_{2}$ or $\mathrm{O}_{2}$ gas into the TEM chamber (though not by adding $\mathrm{Ar}$ gas). This indicates that air composed mainly of $\mathrm{N}_{2}$ and $\mathrm{O}_{2}$ is the essential factor for thermal coarsening of NPG, since the adsorbates created on the gold surface by $\mathrm{N}_{2}$ and $\mathrm{O}_{2}$ molecules promote surface

*Corresponding author, E-mail: s_kuwano@mail.tohoku-gakuin.ac.jp diffusion. We conclude with a discussion of the mechanism of surface diffusion and the effects of the molecular gas on the ligament coarsening of nanoporous gold.

\section{Experimental Procedures}

NPG films were fabricated by chemically dealloying a $\sim 100 \mathrm{~nm}$ thick $\mathrm{Au}_{35} \mathrm{Ag}_{65}$ (at\%) leaf in a 70 vol\% $\mathrm{HNO}_{3}$ solution (Wako Pure Chemical Industries, Osaka, Japan) for $5 \mathrm{~min}$ at $40^{\circ} \mathrm{C}$. The as-prepared samples were then carefully rinsed three times with distilled water $(18.2 \mathrm{MW} \mathrm{cm})$, for 15-20 min per rinse, to remove the residual nitric acid. The ligament/pore structure was measured by a scanning electron microscope (SEM) (JIB-4600F, JEOL, Japan). The average pore size of the as-prepared sample was tailored to within $20-30 \mathrm{~nm}$, according to a previous study. ${ }^{4}$ ) The composition of the NPG film was determined by X-ray energy-dispersive spectroscopy (EDX: Standard, EDAX Inc., Japan) with an attached SEM (S-3400N, Hitachi, Japan). The residual amount of Ag in the NPG was less than 4 at $\%$.

Average nanopore sizes were measured by a rotationally averaged fast Fourier transform (FFT) method. ${ }^{8)}$ This FFT method analyzes the period of black areas (pore) and white areas (ligament) in the SEM images containing 200-300 ligament/pores. Briefly, the SEM images were transformed into FFT power spectra that contain scattering peaks corresponding to the characteristic length scales of the bicontinuous nanostructure. Pore size is - as defined by the equivalent diameters of nanopores or gold ligaments estimated at half of the characteristic length scale.

The NPG films were annealed in a furnace at temperatures ranging from 125 to $400^{\circ} \mathrm{C}$ for up to 10 hours to observe the coarsened ligament/pore structures in air. Additional samples were sealed in glass tubes at a vacuum of $0.4 \sim 1 \times 10^{-2} \mathrm{~Pa}$ for annealing. To understand the underlying mechanisms of the deploying process, we measured the activation energy of 

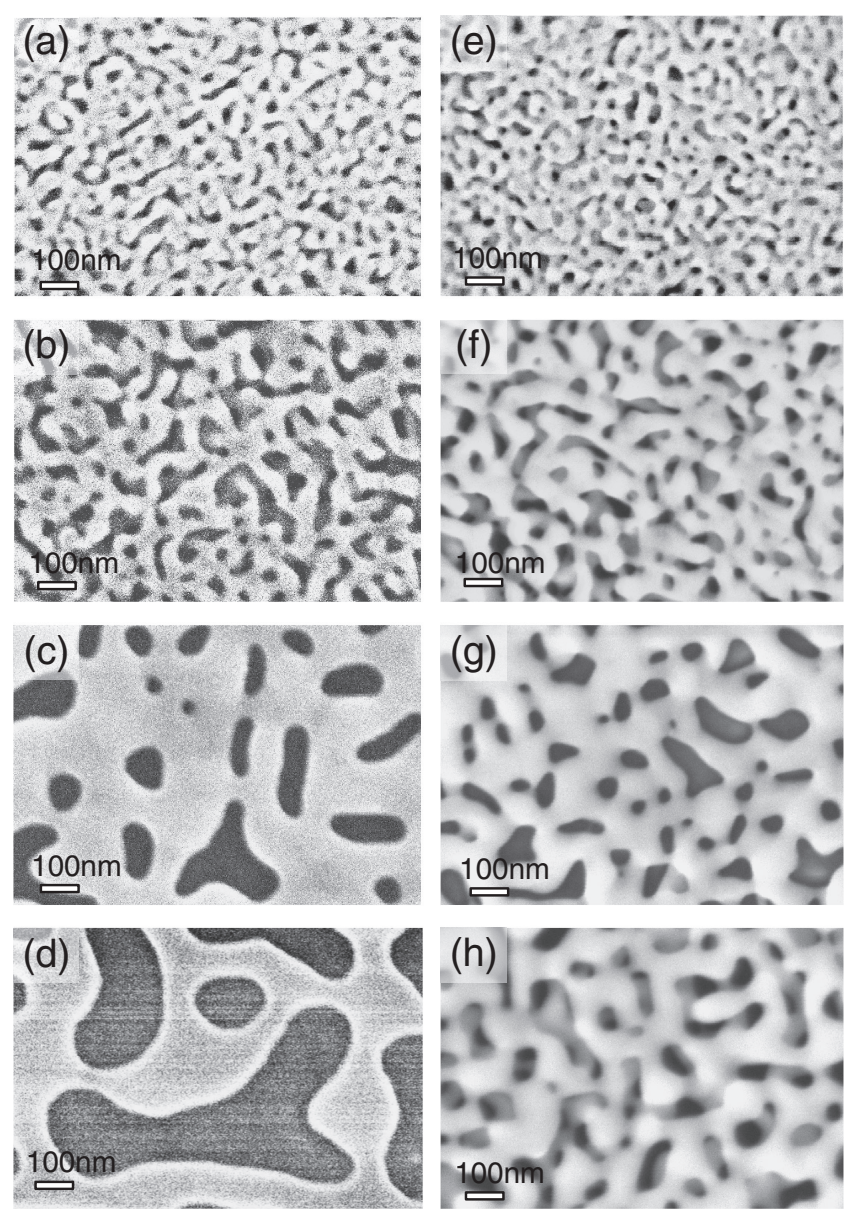

Fig. 1 Plane-view SEM images of NPG annealing for 4 hours at various temperatures. (a) as-prepared, (b) $200^{\circ} \mathrm{C}$, (c) $300^{\circ} \mathrm{C}$, (d) $400^{\circ} \mathrm{C}$ in air, and (e) as-prepared, (f) $200^{\circ} \mathrm{C}$, (g) $300^{\circ} \mathrm{C}$, (h) $400^{\circ} \mathrm{C}$ in vacuum.

thermal coarsening for NPG using log-linear fits of pore size versus annealing times. ${ }^{4}$ )

For in situ observation, the NPG films were transferred onto copper TEM grids, without a carbon supporting film, for subsequent microstructure characterization. For ETEM, we used a $1000 \mathrm{kV}$ JEM-1000K RS TEM (JEOL, Japan) equipped with an environmental cell designed in-house at Nagoya University, Japan. ${ }^{9)}$ The point-to-point resolution of the TEM was $0.15 \mathrm{~nm}$, and the base vacuum pressure was $10^{-5} \mathrm{~Pa}$. We examined NPG samples in pure $\mathrm{N}_{2}(5 \mathrm{~N}), \mathrm{O}_{2}$ $(6 \mathrm{~N})$, and $\operatorname{Ar}(5 \mathrm{~N})$ environments at $400^{\circ} \mathrm{C}$ and over a wide range of total pressures ( 1 to $30 \mathrm{~Pa})$. The current flux of the electron beam was measured using a Faraday gauge ( 0.14 to $\left.0.23 \mathrm{~A} / \mathrm{cm}^{2}\right)$; a single-tilt heating stage, designed for the JEM-1000K RS (Gatan, USA), was also employed.

\section{Results and Discussion}

The as-prepared NPG samples were annealed at 200 to $400^{\circ} \mathrm{C}$ in air or in vacuum $\left(0.4 \sim 1 \times 10^{-2} \mathrm{~Pa}\right)$. Figure 1 (a) through (d) show SEM images of NPG films that were annealed as-prepared at 200,300 , and $400^{\circ} \mathrm{C}$ for 4 hours in air. Figures 1(e) through (h) show the pore structures of the NPG films in vacuum for equivalent annealing temperatures/ times. When annealing in air, pore size tends to be proportional to annealing temperature. However, when

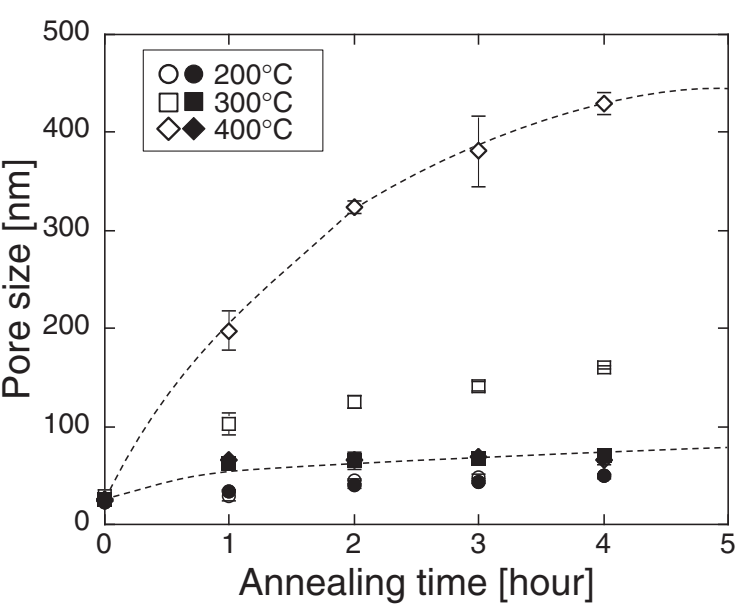

Fig. 2 Evolution of pore size of NPG samples during annealing in air (open symbols) and vacuum (closed symbols). The dotted lines are tracing the conditions of the samples heated at $400^{\circ} \mathrm{C}$.

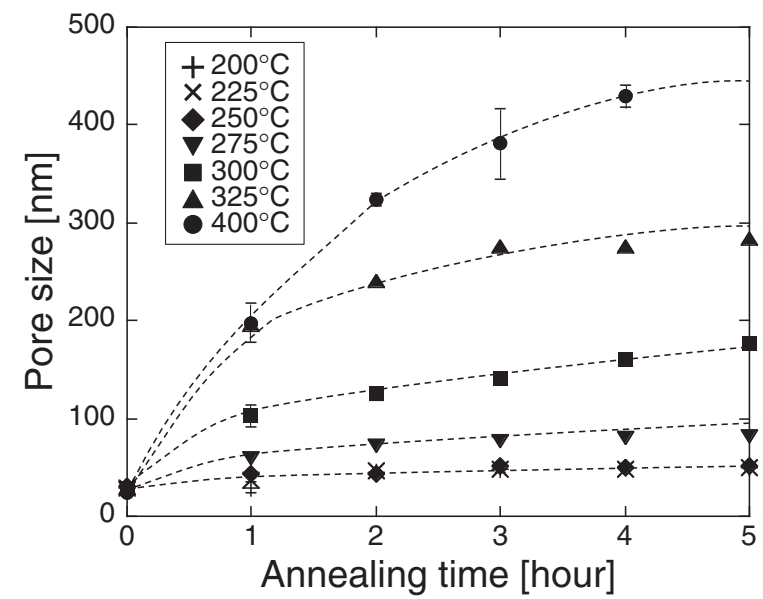

Fig. 3 Evolution of pore size for the NPG samples during annealing at various temperatures in air.

annealing in vacuum, pores size did not increase significantly with temperature.

Figure 2 provides a summary of measured pore sizes with various annealing times and temperatures. Clearly, the coarsening behavior is different in the two environments (in air and in vacuum) at elevated temperatures. Figure 3 details the evolution of pore size in NPG at various temperatures as a function of annealing time in air. For cases above $250^{\circ} \mathrm{C}$, pore size increased dramatically. In contrast, Fig. 4 details the evolution of pore size in vacuum. For cases below $225^{\circ} \mathrm{C}$, the pore size increased continuously, and continued to grow even after 4 hours of annealing. However, when the NPG was heated above $250^{\circ} \mathrm{C}$, the pore size was saturated after 1 hour of annealing, and average pore size was suppressed to less than $60-90 \mathrm{~nm}$, even when subjected to longer annealing times.

The coarsening rate of the nanopores is similar to the isothermal grain growth in polycrystalline materials. ${ }^{4)}$ They estimated the coarsening kinetics by a log-linear fit of pore size versus etching times, i.e.,

$$
d(t)^{n}=k_{0} t \exp \left(\frac{-E}{R T}\right)
$$




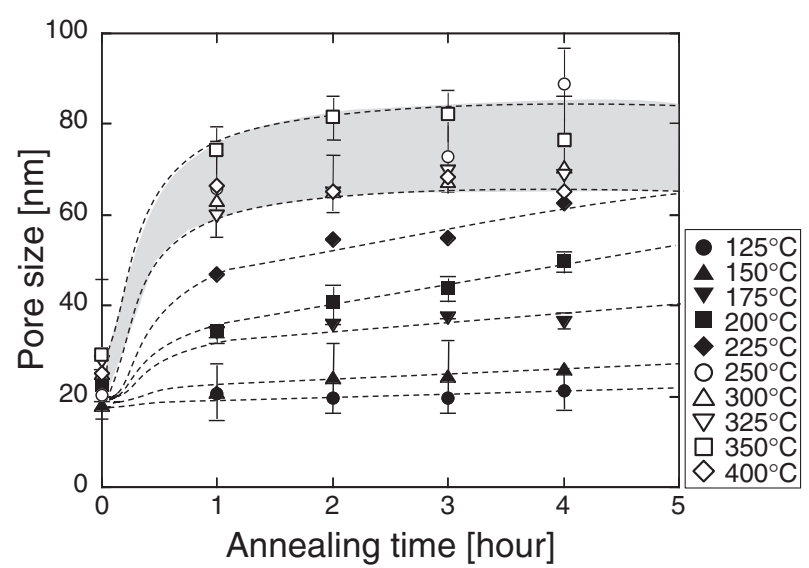

Fig. 4 Evolution of pore size of NPG samples during annealing at various temperatures in vacuum.
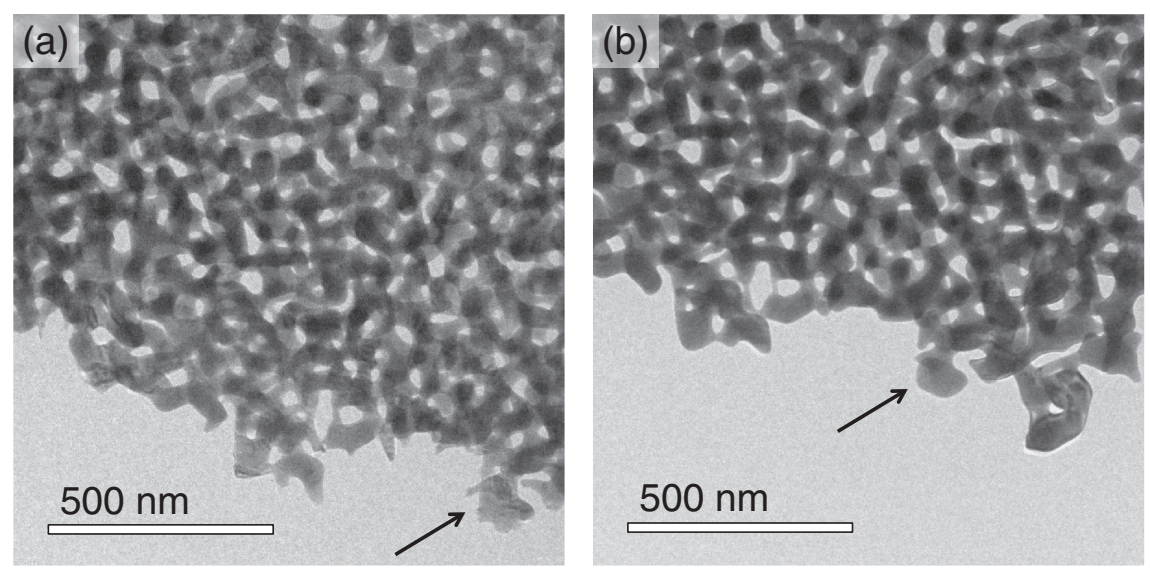

Fig. 6 TEM images of annealing in vacuum $\left(10^{-5} \mathrm{~Pa}\right)$ at $400^{\circ} \mathrm{C}$. (a) Before annealing, (b) after annealing for 5 min. The arrows indicate a specific location in the NPG.

where $d(t)$ is pore size at etching time $t, k_{0}$ is a constant, $n$ is a coarsening exponent, $R$ is the gas constant, $T$ is the annealing temperature in our work (instead of the etching temperature), and $E$ is the activation energy for nanopore formation and coarsening. The coarsening exponent $n$ can be measured by plotting $\ln [d(t)]$ versus $\ln t$. Then, the activation energy for the coarsening of nanoporous gold is based on the linear fit between $\ln \left(d(t)^{n} / t\right)$ and $(R T)^{-1}$.

We then calculated the activation energy of coarsening kinetics during annealing with the same method. From the pore-size plot results in Figs. 2-4, the $n$ values for the coarsening of nanoporous gold were determined to be $4.8 \pm 1.9$ in air and $5.7 \pm 1.4$ in vacuum. These measured $n$ were compatible with the kinetic parameter, 4 , reflecting a coalescence process of roughened metals. ${ }^{10}$ ) We then calculated the activation energy of coarsening kinetics by a $\log$-linear fit of pore size versus annealing times, as shown in Fig. 5. From these results, the activation energy for the thermal coarsening of NPG was measured to be $85.6 \mathrm{~kJ} / \mathrm{mol}$ in air and $182.2 \mathrm{~kJ} / \mathrm{mol}$ in vacuum. This vacuum-annealing activation energy was nearly equal to that of lattice diffusion of gold $(174.6 \mathrm{~kJ} / \mathrm{mol}){ }^{11,12)}$ We presume that this difference arose as follows: In air, the surface adsorption of gas molecules promotes the surface diffusion of $\mathrm{Au}$ adatoms on NPG, which is the dominant mechanism of thermal

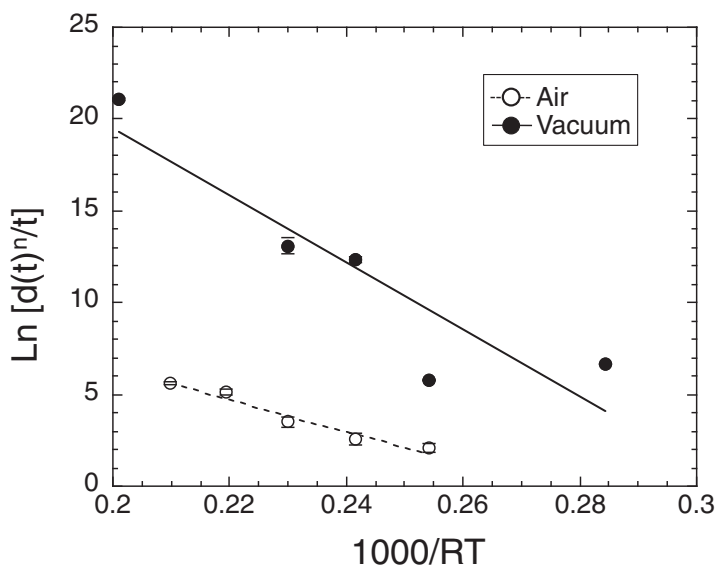

Fig. 5 Estimation of the activation energy for the annealing of NPG in air and in vacuum. coarsening in air (i.e. gas). On the contrary, for annealing in vacuum, gas molecules have a negligible effect on the surface diffusion of Au. Therefore, lattice diffusion - not surface diffusion - is dominant in the coarsening of NPG in vacuum annealing.

Next, we investigated the thermal coarsening of NPG by ETEM to distinguish the effects of specific types of gas. The samples were annealed at $400^{\circ} \mathrm{C}$ either in vacuum or pure $\mathrm{N}_{2}, \mathrm{O}_{2}$, or Ar gas in the TEM chamber. Figure 6 shows the microstructure of a sample annealed at $400^{\circ} \mathrm{C}$ in vacuum $\left(10^{-5} \mathrm{~Pa}\right)$; the arrows mark a specific position before and after annealing for comparison. Interestingly, the annealed sample in vacuum did not show any significant coarsening, which supports our conclusions from the ex situ observations. Next, we heated another sample to $400^{\circ} \mathrm{C}$ in vacuum, verified minimal structural changes, and then injected an $\mathrm{N}_{2}$ gas flow of up to $30 \mathrm{~Pa}$ into the TEM chamber. Figure 7(a) shows the sample morphology before injecting the $\mathrm{N}_{2}$ flow, with the other images showing the coarsening process over the same area for a $\mathrm{N}_{2}$ flow of (b) $7 \mathrm{~min}$, (c) $10 \mathrm{~min}$, and (d) $15 \mathrm{~min}$. Here, the ligament morphology changed significantly.

For annealing under $\mathrm{O}_{2}$ gas flow, we repeated the same procedure, which resulted in the same tendency observed with $\mathrm{N}_{2}$. Figure 8 shows the morphological changes under $\mathrm{O}_{2}$ gas flow $(6 \mathrm{~Pa})$ at $400^{\circ} \mathrm{C}$. Most ligaments exhibit agglomer- 

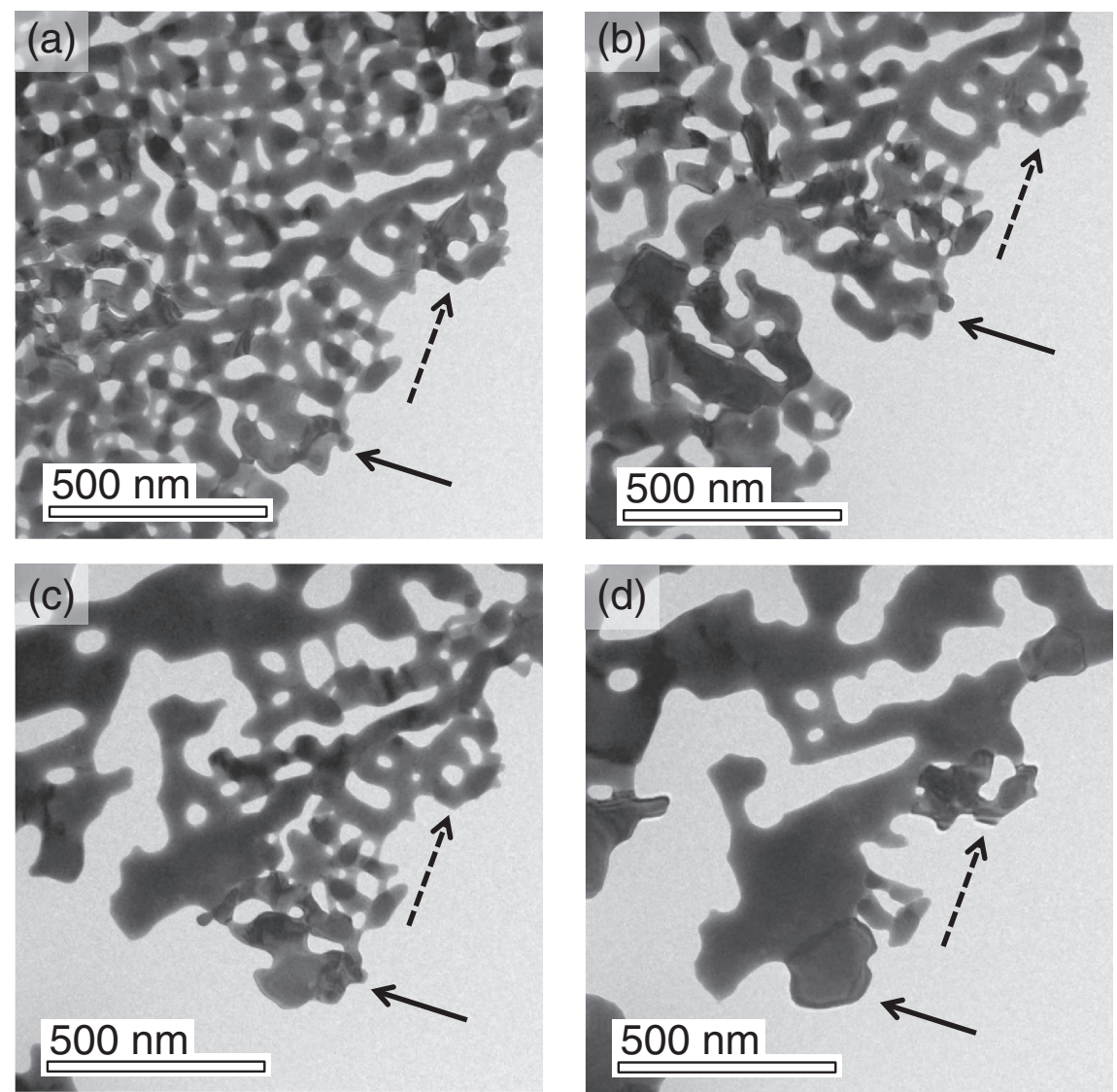

Fig. 7 TEM images of NPG annealed at $400^{\circ} \mathrm{C}$. (a) In vacuum, and after initiating $\mathrm{N}_{2}$ gas flow (30 Pa) for (b) $7 \mathrm{~min}$, (c) $10 \mathrm{~min}$, and (d) $15 \mathrm{~min}$.
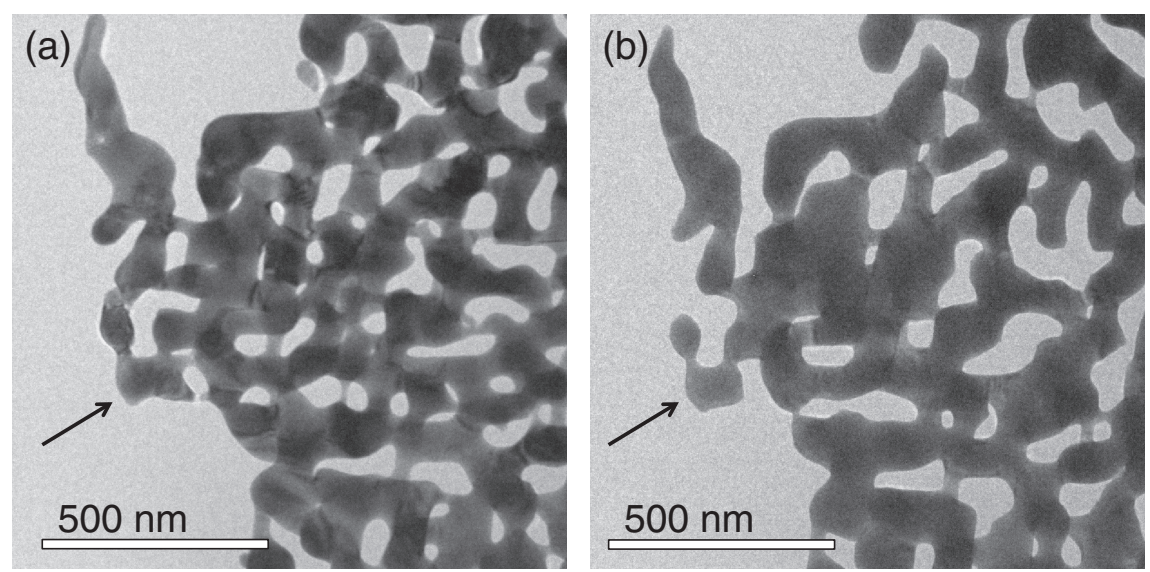

Fig. 8 TEM images of NPG annealed at $400^{\circ} \mathrm{C}$. (a) In vacuum, (b) after 5 min of $\mathrm{O}_{2}$ gas flow (6 Pa).

ation during annealing. However, with an Ar gas flow (30 Pa) at $400^{\circ} \mathrm{C}$ - to test for any effects from gas flow itself ${ }^{13)}$ - we observed only minimal structural change (Fig. 9).

In sum, pore coarsening at elevated temperatures was observed with $\mathrm{N}_{2}$ or $\mathrm{O}_{2}$, but not with Ar. This indicates that non-inert gas species are effective for thermal coarsening. In particular, the coarsening under pure $\mathrm{N}_{2}$ flow was exceptional because $\mathrm{N}_{2}$ is inactive with gold in general. We thus pose an open question of whether the triple bonds of $\mathrm{N}_{2}$ were partially severed and activated on the NPG. Regardless, this result originates from the difference in adsorption processes for inert monoatoms (e.g. Ar) and chemically active diatoms (e.g. $\mathrm{N}_{2}, \mathrm{O}_{2}$ ). An inert Ar molecule was able to adsorb physically (physisorption) on the surface of NPG, while the chemically active $\mathrm{N}_{2}$ and $\mathrm{O}_{2}$ molecules were able to adsorb chemically. Since $\mathrm{N}_{2}$ and $\mathrm{O}_{2}$ have high electronegativity, $\mathrm{N}_{2}$ and $\mathrm{O}_{2}$ are attracted to electron density on a surface. This polarity between adsorbed gas molecules and top-layer $\mathrm{Au}$ atoms results in a reduced bonding energy between these toplayer $\mathrm{Au}$ atoms and those below. Consequently, the topmost $\mathrm{Au}$ atoms have a tendency to leave step edges and diffuse over an NPG surface as adatoms. On a related note, Fujita et al. observed that pore size increased with the exothermic reaction of $\mathrm{CO}$ oxidation at room temperature. On the 

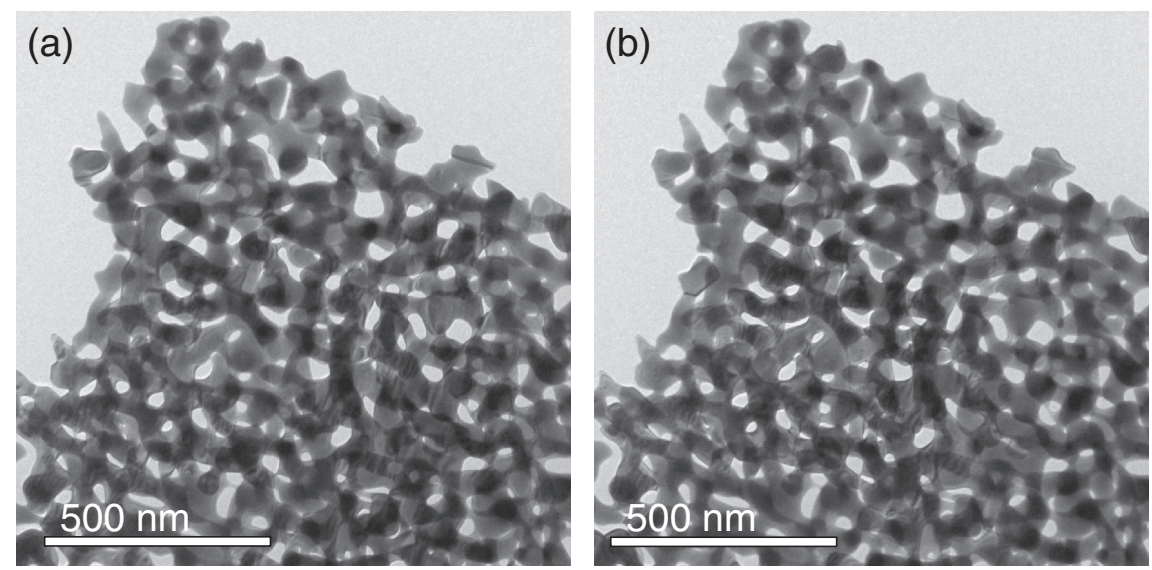

Fig. 9 TEM images of NPG annealed at $400^{\circ} \mathrm{C}$. (a) In vacuum, (b) after 5 min of Ar gas flow (30 Pa).

contrary, pore size was not affected by endothermic reactions such as CO decomposition. ${ }^{14)}$ Therefore, both the chemical activation/reaction of adsorbate and the thermal energy from either an exothermic reaction or an external heating source are the effective driving forces for ligament/pore coarsening of NPG.

\section{Conclusion}

In this study, to investigate the environmental factors governing NPG ligament/pore coarsening, NPG samples were annealed in vacuum and air, as well as in $\mathrm{N}_{2}, \mathrm{O}_{2}$, and $\mathrm{Ar}$ gas. We observed significant pore coarsening in air and in chemically active diatomic gas $\left(\mathrm{N}_{2}\right.$ and $\left.\mathrm{O}_{2}\right)$, but not in vacuum and inert monoatomic gas (Ar). We concluded that both the chemical activation of adsorbate and thermal energy are environmental requirements for ligament/pore coarsening of NPG.

\section{Acknowledgements}

This work was primarily sponsored by KAKENHI 25708036, and was performed under the Inter-university Cooperative Research Program of the Institute for Materials Research, Tohoku University (Proposal No. 11K0072, 12K0100, 13G0042, 14G0031). We also acknowledge the support from the Advanced Characterization Nanotechnology Platform of the High Voltage Electron Microscope Laboratory of Nagoya University and the Hi-tech Research Center of Tohoku Gakuin University. Additionally, we acknowledge Teruyoshi Awano for his constant technical support.

\section{REFERENCES}

1) Y. Ding and M. W. Chen: MRS Bull. 34 (2009) 569-576.

2) A. Wittstock, V. Zielasek, J. Biener, C. M. Friend and M. Baumer: Science 327 (2010) 319-322.

3) J. Biener, A. Wittstock, L. A. Zepeda-Ruiz, M. M. Biener, V. Zielasek, D. Kramer, R. N. Viswanath, J. Weissmuller, M. Baumer and A. V. Hanza: Nature Mater. 8 (2009) 47-51.

4) L. H. Qian and M. W. Chen: Appl. Phys. Lett. 91 (2007) 083105.

5) F. Kertis, J. Snyder, L. Govada, S. Khurshid, N. Chayen and J. Erlebacher: JOM 62 (2010) 50-56.

6) Y. Sun, S. A. Burger and T. J. Balk: Philos. Mag. 94 (2014) 1001-1011.

7) T. Fujita, P. Guan, K. McKenna, X. Lang, A. Hirata, L. Zhang, T. Tokunaga, S. Arai, Y. Yamamoto, N. Tanaka, Y. Ishikawa, N. Asao, Y. Yamamoto, J. Erlebacher and M. W. Chen: Nature Mater. 11 (2012) $775-780$.

8) T. Fujita and M. W. Chen: Jpn. J. Appl. Phys. 47 (2008) 1161-1163.

9) N. Tanaka, J. Usukura, M. Kusunoki, Y. Saito, K. Sasaki, T. Tanji, S. Muto and S. Arai: Microscopy 62 (2013) 205-215.

10) J. M. Dona and J. Gonzalez-Velasco: J. Phys. Chem. 97 (1993) 4714 4719.

11) S. M. Makin, A. H. Rowe and A. D. LeClaire: Proc. Phys. Soc. B 70 (1957) 545-552.

12) W. C. Mallard, A. B. Gardner, R. F. Bass and L. M. Slifkin: Phys. Rev. 129 (1963) 617-625.

13) D. L. Olson, H. R. Patil and J. M. Blakely: Scr. Metall. 6 (1972) 229_240.

14) T. Fujita, T. Tokunaga, L. Zhang, D. Li, L. Y. Chen, S. Arai, Y. Yamamoto, A. Hirata, N. Tanaka, Y. Ding and M. W. Chen: Nano Lett. 14 (2014) 1172-1177. 